\title{
BUCKLING OF STEPPED BEAMS RESTING ON AN ELASTIC FOUNDATION
}

\author{
Krzysztof Kuliński, Jacek Przybylski \\ Institute of Mechanics and Machine Design Foundations, Czestochowa University of Technology \\ Czestochowa, Poland \\ krzysztku@gmail.com,j.przybylski@imipkm.pcz.pl
}

\begin{abstract}
The influence of structural parameters of a stepped beam with two ends fixed and resting on Winkler foundation on its buckling critical force has been discussed in this paper. The structure inhomogeneity results from two piezoceramic plates perfectly bonded at the top and bottom surface of the beam. For the performed analysis five different supports of beam ends which prevent longitudinal displacements have been adopted. Numerical analysis has been divided into two parts. The first part concerns the influence of the system geometry on its critical force, whereas in the second part, a modification of the buckling load resulting from the electric field applied to the piezosegment has been investigated.
\end{abstract}

Keywords: buckling, critical force, stepped beam, Winkler foundation, piezoelectricity, piezoceramics

\section{Introduction}

The stability of beams and columns under axial compressive force with different ends support, stepped cross-sections and resting on elastic foundations has been the subject of interest of many researchers. In their studies the problem has been formulated by using both classical mathematical methods and finite element analysis. The use of different elastic foundations have been presented by Kerr [1], where Winkler, Pasternak, Vlaslov, Filonenko-Borodich foundation have been selected for the analysis. In the case of the Winkler foundation, reaction forces are proportional to the deflection of a beam at each point of contact and the foundation characteristics are modelled by adopting the system of fixed linear springs. The constant of proportionality of these springs is known as the subgrade modulus. Jančo [2] used the finite element method to evaluate the buckling load of a pinned-pinned beam and to compare the obtained results to the exact solution. The analysis showed some discrepancies, especially for the beams where were used less than fifteen finite elements. Wang et al. [3] have focused their attention on the static buckling characterized by bifurcation. The authors have solved stability problems for columns, arches, plates and shells. The obtained results concerned, among others, 
the objects with internal hinges, rotational restrained joints and an elastic foundation. Boundary regions of stability for a rod under a two-parameter potential and non-potential loads have been determined by Glabisz [4]. Using classical formulation, the author has proven that simultaneous application of independent potential and non-potential loads to the beam results in an increased critical force which can be obtained in the case, when only spatially determined forces act in the system. The finite element method has been applied by Dudzik and Obara [5] to examine the stability of the Timoshenko beam resting on a two-parameter elastic foundation. In that paper the exact stiffness matrix of the basic beam element has been derived and the equation for the critical load has been specified. It has been stressed in the stated conclusions that the effect of elastic foundation on the buckling load decreases with the reduction of beam slenderness. Fazelzadeh and Kazemi-Lari [6] have considered the stability of a partially loaded Leipholz column carrying lumped mass and resting on an elastic Winkler foundation. The role of elastic foundation modulus, ratio of the lumped mass to the column's mass, position of the lumped mass and four different types of load distribution have been examined. Eryilmaz et al. [7] have presented a study in the buckling instability for Euler columns with continuous elastic restraint on the basis of homotopy analysis method (HAM). Results concerned the effect of five different ways of beam support and the effect of the elastic restraint coefficient on the first and second modes of buckling. The authors have documented an excellent agreement between results obtained on the basis of HAM and other analytical methods.

Application of piezoceramic actuators and their effect on the stability and natural frequency of slender beams has been studied by Przybylski [8]. The author has considered axially compressed column, divided into three segments, with both ends sliding in the direction perpendicular to the undeflected axis of the system. The middle segment consisted of two piezoelectric actuators colocally and perfectly bonded to the host column. A weakening hinge, but strengthened with a spring support between the second and third segments, has been taken into consideration. The von Karman theory has been utilised to describe the strain-displacement relation. A broader literature overview and wider area of study of beams with integrated ceramic piezoactuators excluding elastic foundation can be found in [9].

In this paper the influence of structural parameters of the stepped beam resting on the Winkler foundation on its buckling critical load is studied. Five different ends that support eliminating longitudinal displacement are taken into consideration. The change in the beam's cross-section is an effect of two piezoceramic patches mounted collocally at the specified location on the top and bottom surfaces of the beam. It is assumed that adhesive layers connecting mentioned elements are negligibly small and the bonding remains perfect at the entire length of the connection. One of the main purposes of this study is to investigate the effect of the electric field application to the piezoceramic patches on the stability of a considered beam. 


\section{Formulation of the problem}

A scheme of a pinned-pinned three-segmented beam resting on Winkler foundation of modulus $k$ is shown in Figure 1. A prescribed end displacement $\delta$ of one support creates axial force $P$ of a value which is determined from Hooke's law. The beam along its piezosegment is influenced by the electric field of vector $\boldsymbol{E}$.

In the analysis five different supports of beam ends preventing longitudinal displacements are used: clamped-clamped (C-C), clamped-pinned (C-P), pinned-pinned (P-P), clamped-guided (C-G), pinned-guided (P-G).

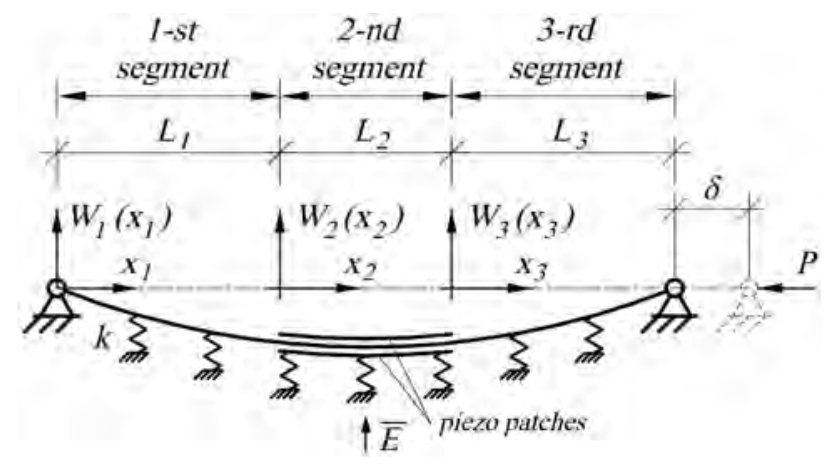

Fig. 1. Scheme of the pinned-pinned beam with centrally located piezosegment

For all analyzed models three different localisations of piezosegment have been considered as shown in Figure 2 for a C-P beam:

a)

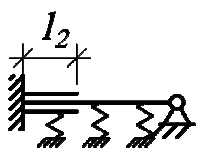

b)

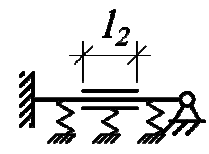

c)

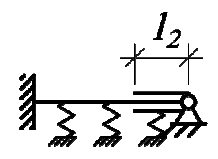

Fig. 2. Piezosegment localizations: a) at the left-hand side support, b) central localization, c) at the right-hand side support

In order to formulate and analyze the problem, the following assumptions have been adopted:

- before load application the beam is rectilinear and made of homogeneous linearly elastic isotropic material model,

- three different non-dimensional elastic foundation coefficients have been used for all considered models,

- system's cross-section is rectangular; width $b$ is greater than height of piezosegment $\left(2 h_{p}+h_{b}\right)$ according to the following relation $b /\left(2 h_{p}+h_{b}\right) \geq 2$,

- piezoceramic actuators are made of homogeneous elastic and transversely isotropic material model and are perfectly bonded to the beam along the whole length,

- the adhesive layer thickness is treated as negligibly small, 
- exactly the same dimension relations are used in all models,

- both upper and bottom piezoelements are under a uniform constant electric field.

The constitutive equation describing the piezoelectric material model in onedimensional problems has the following form:

$$
\sigma=E_{p} \varepsilon(x) \pm e_{31} V / h_{p}
$$

where: $E_{p}$ denotes Young's modulus of piezoelectric material model, $\varepsilon(x)$ is the strain along the beam, $e_{31}$ is the piezoelectric constant, $V$ is the driving voltage. The residual force equation for a $n$-segmented beam with fixed ends in axial direction has been derived by Przybylski [9]. That force for the considered three-segmented system takes the form:

$$
F_{r}=F\left[1+\eta\left(\frac{L}{L_{2}}-1\right)\right]^{-1}
$$

where: $F= \pm 2 b e_{31}$ denotes piezoelectric tensile or compressive force induced by a pair of piezo-patches, $\eta$ is the coefficient identified as relation of piezosegment axial stiffness to the beam axial stiffness.

The non-dimensional governing equation for $i$-th segment including a residual force term which is activated by the electric field applied to the system is as follows [9]:

$$
\frac{\partial^{4} w_{i}\left(\xi_{i}\right)}{\partial \xi_{i}^{4}} \pm \varphi_{i} p_{0}^{2} \frac{\partial^{2} w_{i}\left(\xi_{i}\right)}{\partial \xi_{i}^{2}}+\beta w_{i}\left(\xi_{i}\right)=0 \text { where } p_{0}^{2}=p_{u}^{2}+f_{r}^{2}
$$

Here $p_{u}^{2}$ is the force parameter resulting from a prescribed axial support displacement and $f_{r}^{2}$ is the dimensionless parameter of the residual force expressed by equation (2).

All dimensionless parameters have been obtained through the substitutions:

$$
\begin{gathered}
w_{i}\left(\xi_{i}\right)=\frac{W_{i}\left(x_{i}\right)}{L}, \quad \xi_{i}=\frac{x_{i}}{L}, \quad p_{0}^{2}=\frac{P_{0} L^{2}}{E_{b} I_{b}}, \quad p_{u}^{2}=\frac{P_{u} L^{2}}{E_{b} I_{b}}, \quad f_{r}^{2}=\frac{F_{r} L^{2}}{E_{b} I_{b}}, \\
l_{i}=\frac{L_{i}}{L}, \quad \varphi_{i}=\left(1+r_{m}\right)^{-\frac{1}{2}\left(j^{2 i}+1\right)}, \quad \beta=\frac{k L^{4}}{E_{b} I_{b}}, \quad r_{m}=\frac{E_{p} I_{p}}{E_{b} I_{b}}, \quad j=\sqrt{-1}, \\
\eta=1+\alpha_{1} \frac{A_{p}}{A_{b}}, \quad \alpha_{1}=\frac{E_{p}}{E_{b}}
\end{gathered}
$$

where: $W_{i}\left(x_{i}\right)$ - is the transversal displacements, $\beta$ is the elastic foundation coefficient, $E_{b} I_{b}, E_{p} I_{p}$ express the beam and the piezoelectric patches bending stiffness, 
respectively, and $A_{p}, A_{b}$ refer to the piezoceramic stripes and the beam crosssection area, respectively.

As the solution and analysis of the problem need to cover all types of supports preventing longitudinal displacements of the beam ends, the boundary conditions have been divided into two groups, in which:

a) the first group contains continuity boundary conditions which describe the equality of transverse displacements, slopes, bending moments and shear forces between the first and second segments and between the second and third segments:

$$
\begin{aligned}
& \left.w_{i}\left(l_{i}\right)\right|_{\xi_{i}=l_{i}}=\left.w_{i+1}\left(\xi_{i+1}\right)\right|_{\xi_{i+1}=0}=\left.w_{i}^{I}\left(l_{i}\right)\right|_{\xi_{i}=l_{i}}=\left.w_{i+1}^{I}\left(\xi_{i+1}\right)\right|_{\xi_{i+1}=0}=0 \\
& \left.\left(1+r_{m}\right)^{\frac{1}{2}\left(j^{2 i}+1\right)} w_{i}^{R n}\left(\xi_{i}\right)\right|_{\xi_{i}=l_{i}}=\left.\left(1+r_{m}\right)^{\frac{1}{2}\left(j^{2(i+1)}+1\right)} w_{i+1}^{R n}\left(\xi_{i+1}\right)\right|_{\xi_{i}+1=0}=0
\end{aligned}
$$

where: $i=1,2$ and $R n=I I, I I I$ are the Roman numerals denoting the order of the derivative with respect to the space variable $\xi$.

b) the second group specifies the boundary conditions for all beams. For the pinned-pinned (P-P) system from Figure 1 the boundary conditions are:

$$
w_{1}(0)=w_{1}^{I I}(0)=w_{3}\left(l_{3}\right)=w_{3}^{I I}\left(l_{3}\right)=0
$$

where: For a clamped support both the displacement $w$ and the slope $w^{I}$ are equal to zero; For a guided support both the slope $w^{I}$ and the shear force $w^{I I I}$ are equal to zero. For a system with a mixed type of supports, adequate boundary conditions have to be chosen from those described above.

\section{Solution}

The general solution of equation (3), can be demonstrated as follows:

$$
w_{i}\left(\xi_{i}\right)=A_{i} \cosh \left(\gamma_{1} \xi_{i}\right)+B_{i} \sinh \left(\gamma_{1} \xi_{i}\right)+C_{i} \cosh \left(\gamma_{2} \xi_{i}\right)+D_{i} \sinh \left(\gamma_{2} \xi_{i}\right), i=1,2,3
$$

Coefficients $\gamma_{1}$ and $\gamma_{2}$ are expressed as:

$$
\gamma_{1}=\sqrt{\frac{ \pm \varphi_{i} p_{0}^{2}-\sqrt{\varphi_{i} p_{0}^{4}-4 \beta}}{2}}, \quad \gamma_{2}=\sqrt{\frac{ \pm \varphi_{i} p_{0}^{2}+\sqrt{\varphi_{i} p_{0}^{4}-4 \beta}}{2}}
$$

Substituting equation (7) into the adequate set of boundary conditions (5), (6) corresponding to the specified supports, one obtains a system of twelve homogeneous linear equations with respect to unknown integration constants $A_{i}, B_{i}, C_{i}$ and $D_{i}$ 
$(i=1,2,3)$. Equating to zero the determinant of matrix coefficients of that system one derives a transcendental equation from which the set of successive critical loads can be numerically determined. Each of the buckling modes can be obtained after introduction of a particular eigenvalue into the normalized eigenfunction expressed by equation (7).

\section{Numerical results}

\subsection{The influence of geometry on the critical buckling load}

Figures 3-7 show the non-dimensional buckling load as a function of piezosegment length for different localization of piezoelements and three different elastic foundation coefficients for: clamped-clamped beam (Fig. 3), clamped-pinned beam (Fig. 4), pinned-pinned beam (Fig. 5), clamped-guided beam (Fig. 6), pinnedguided beam (Fig. 7), respectively.

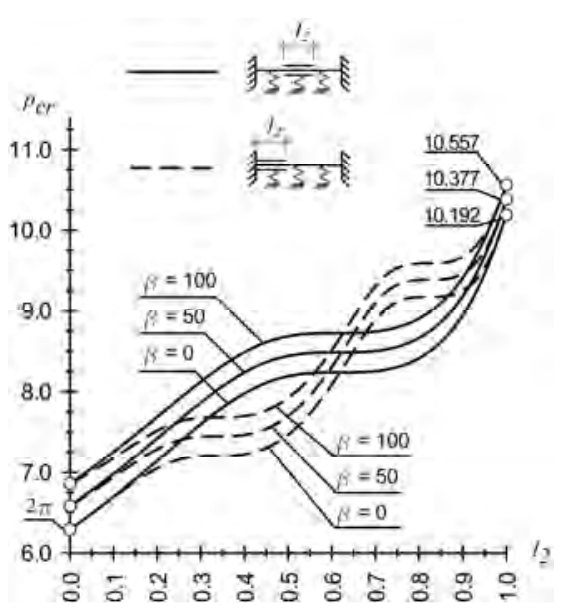

Fig. 3. Critical buckling load versus the piezosegment length for clamped-clamped beam (C-C)

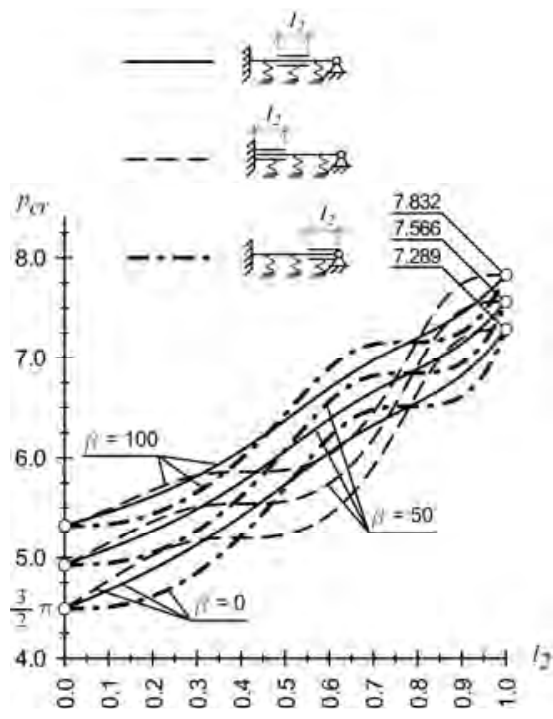

Fig. 4. Critical buckling load versus the piezosegment length for clamped-pinned beam (C-P)

Small icons of beams included in the figures visualize the type of supports and localisation of the piezosegment. Additionally, curves plotted with full lines correspond to the system with piezosegment centrally mounted, dashed lines denote piezoelements placed by the left-hand side support and dash-dot lines indicate piezosegment localized by the right-hand support. If the system has got two different end supports, the position of piezoplates at left and right end of beam is investigated. 


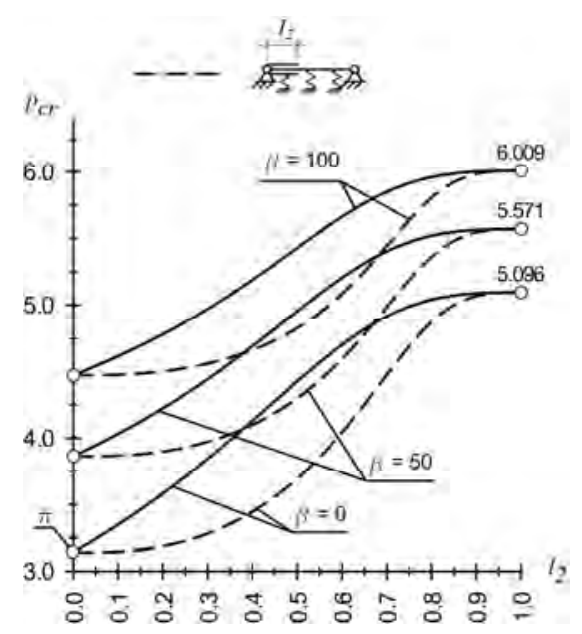

Fig. 5. Critical buckling load versus the piezosegment length for pinned-pinned beam (P-P)
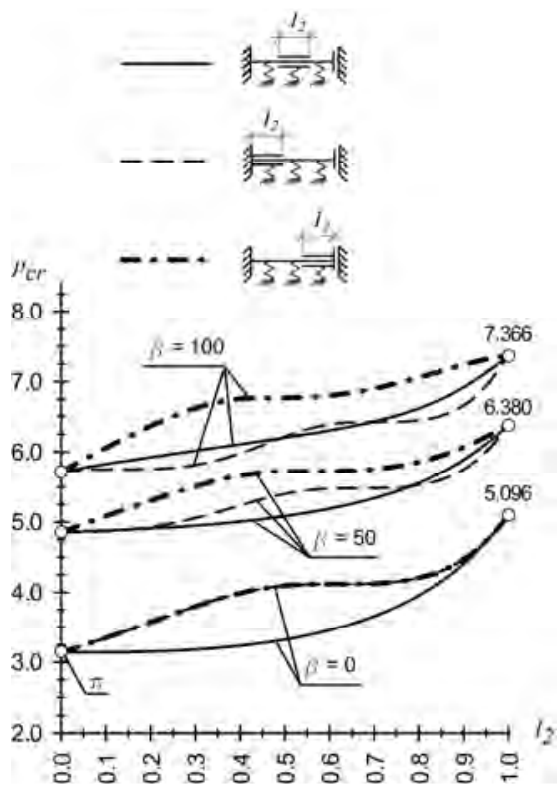

Fig. 6. Critical buckling load versus the piezosegment length for clamped-guided beam (C-G)

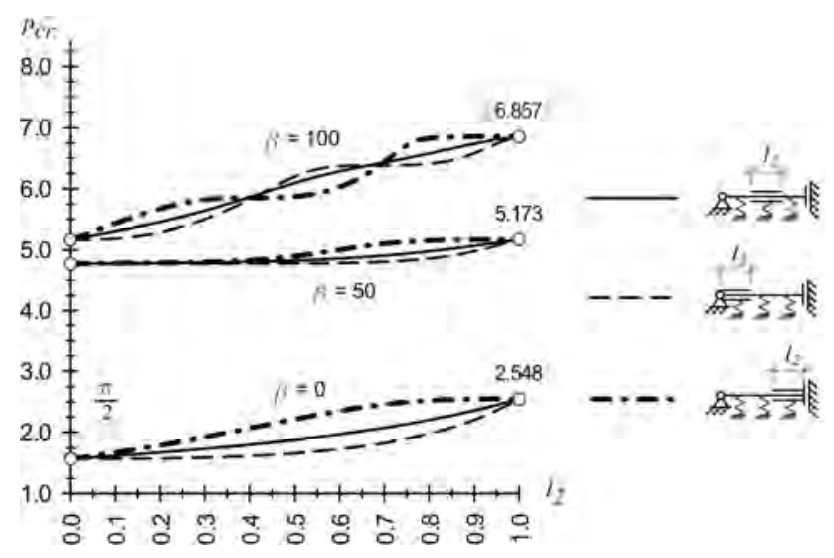

Fig. 7. Critical buckling load versus the piezosegment length for pinned-guided beam (P-G)

Comparing systems from Figures 3-7, one may notice that the highest critical loads for any type of supports exist when the piezosegment is stretched along the entire beam (for $l_{2}=1.00$ ). It is also observed that the greater the foundation modulus, the greater the buckling capacity. The position of piezosegment does not act explicitly on the critical load especially for beams with different supports at their ends. In such cases the values of buckling force change with the length of piezosegment and the appropriate ranges of $l_{2}$ can be determined within which 
the highest critical load appears for a particular localization of piezosegment (cf. Figs. 4 and 7).

One may characterize supports taken into consideration in this study by comparing them in regard to restrictions on the motion. It is known that a classic pin support does not exert any resistance for rotation, whereas a clamped support disables any rotation of the beam axis at the point of its fixing. Both supports prevent against translation in any direction, while a guided support permits vertical translations. Analysing Figures 3-7, it can be stated that the elastic foundation has greater impact on the critical buckling load when the external supports of the beam, despite constrains, allow for a certain type of displacement. This dependency can be studied e.g. by comparing Figures 3 and 7 for $l_{2}=1.0$. Here the absolute difference in the critical load obtained for $\beta=0$ and $\beta=50$ for the beam with both ends clamped is 0.185 (Fig. 3), whereas for the pinned-guided beam (Fig. 7) that absolute difference is as great as 4.309.

In the case where the piezoceramic patches length is equal to zero (prismatic beam), the obtained numerical results can be compared with those presented in [7].

\subsection{Modification of the critical buckling load by the piezoelectric actuation}

The piezoelectric actuators, bonded to the upper and bottom surfaces of a beam, transfer the tensile or compressive force according to the applied electric field when both ends of the beam are constrained against longitudinal displacements. In order to investigate the influence of piezoactuation on the critical load, the nondimensional value of piezoelectric force parameter $f$ have been chosen:

$$
f=\sqrt{\frac{P_{c r}^{(h)}}{E_{b} I_{b}}} \cdot L=\Pi
$$

Value $\pi$ is the non-dimensional buckling force parameter for a uniform beam with both hinged ends. Although all calculations can be made for theoretical models specified above, an analysis of the practical model should also be demonstrated. To verify the applied electric field necessary to generate the piezoelectric force $(f=\pi)$ the beam of $L=600 \mathrm{~mm}$ have been selected. The physical properties of the materials used are given in Table 1. The piezoceramic material P-41 (Annon Piezo Technology Limited Co. [10]) and the aluminium material for the beam have been adopted to the performed analysis.

The critical buckling load for the mentioned beam on the basis of equation (9) is equal to $86.36 \mathrm{~N}$, hence an individual piezoceramic actuator should generate a force equal to $43.18 \mathrm{~N}$. To do that, the necessary voltage of the applied electric field should reach the value of $259.08 \mathrm{~V}$. In accordance to the producer recommendation, the maximal applied voltage without risk of depolarisation of piezoceramics should be lower than $1000 \mathrm{~V}$. Based on the above considerations, the non-dimensional piezoelectric force $(f=\pi)$ can be safely treated as a control value for actuation purposes in the considered system. 
Material properties of the beam and piezosegment

\begin{tabular}{|c|c|c|}
\hline Property & Beam & Piezo-patches \\
\hline Material model & Aluminium & Piezoceramic P-41 \\
\hline$E[\mathrm{GPa}]$ & 70.00 & 83.33 \\
\hline$d_{31}[\mathrm{C} / \mathrm{N}]$ & - & $1.00 \cdot 10^{-10}$ \\
\hline$h[\mathrm{~mm}]$ & 3.00 & 0.50 \\
\hline$b[\mathrm{~mm}]$ & 20.00 & 20.00 \\
\hline$\rho\left[\mathrm{kg} / \mathrm{m}^{3}\right]$ & 2720 & 7450 \\
\hline$U_{\max }[\mathrm{V} / \mathrm{mm}]$ & - & 2000 \\
\hline
\end{tabular}

A prestress created by the piezoelectric force can be treated as an introduction of the compressive or tensile force to the system to counteract the stress that result from an external load. Hence the piezoelectric actuation is a tool for modification of the critical buckling load parameter. Physical and geometrical properties of a piezoelement such as its width, length, thickness and type of piezoceramic material determine not only the maximum value of the electric field but also affect the modification range of the buckling load. The change in the buckling force presented for growing values of the piezosegment length is an effect of actuation by piezoelectric force $f= \pm \pi$.

In Figures 8-10 a modification range of the non-dimensional critical buckling load for a pinned-pinned beam, with three different elastic foundation coefficient parameters, e.a. $\beta=0,50$ and 100 for the centrally located piezosegment has been presented. A full line corresponds to the case when the residual force is equal to zero, a dashed line is relevant to the case when the piezoelectric force compresses the system, whereas a dot-dashed line denotes the system's stretching. It should be noted here, that according to equation (2), the residual piezo-force depends not only on the piezoelectric force but also on the piezosegment to the beam length relation as well as on the axial stiffnesses quotient of the piezosegment to that of the beam.

Considering results from Figures 8-10 for tensile and compressive piezoelectric force obtained for P-P beam it can be noticed that the buckling force modification range increases together with the piezosegment length. Nevertheless, the greater the foundation modulus, the smaller the influence of the piezoactuation (Fig. 11). Comparing the results of percentage modification after generation of the tensile piezoelectric force for the beam with piezosegment of length $l_{2}=1.00$ for $\beta=0$ and $\beta=50$ (Fig. 11), one can notice that the critical load is reduced by $2.67 \%$, while between $\beta=0$ and $\beta=100$ it decreases by $4.63 \%$. Generally, the smaller the foundation parameter, the greater the influence of piezoactuation on the buckling capacity of the system. 


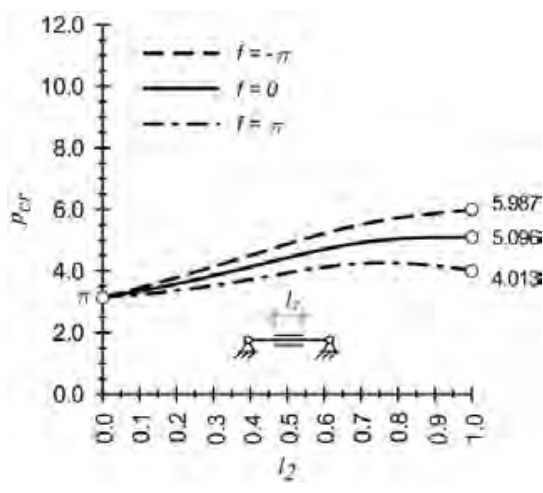

Fig. 8. Modification range of the critical buckling load for different piezosegment length (P-P beam without foundation, $\beta=0)$

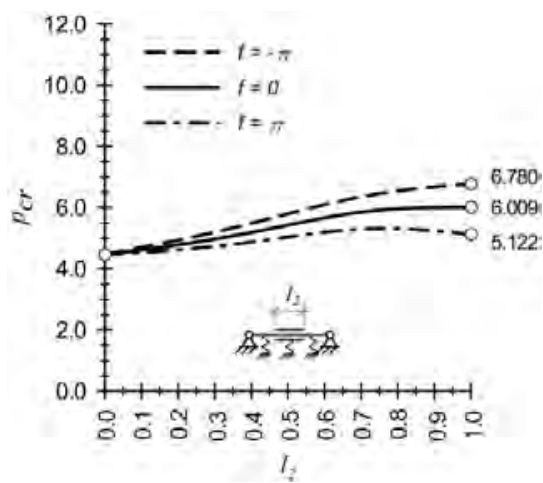

Fig. 10. Modification range of the critical buckling load for different piezosegment length and elastic foundation coefficient

$$
\beta=100 \text { (P-P beam) }
$$

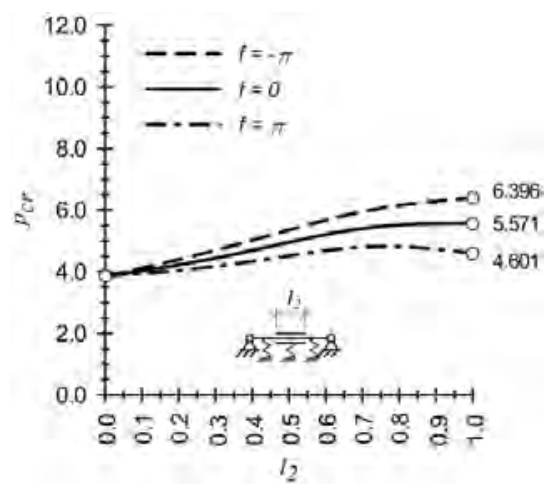

Fig. 9. Modification range of the critical buckling load for different piezosegment length and elastic foundation coefficient $\beta=50$ (P-P beam)

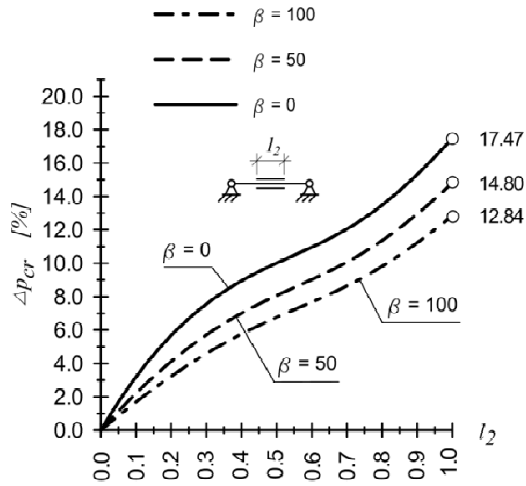

Fig. 11. Percentage range of critical buckling load modification through piezoactuation for different piezosegment length and elastic foundation coefficients (P-P beam)

Hetenyi [11] proved that a simply supported uniform prismatic beam resting on the Winkler foundation buckles with different modes which may be either symmetric or antisymmetric which depends on the foundation parameter. The approach presented in this work makes it possible to find the correct mode shape for a minimal buckling load. The applied numerical procedure leads to computation of the first eigenvalue from the transcendental equation and respective eigenmode. Taking into account the research done by Hetenyi [11], the regions of zeroand one-node modes with regard to elastic foundation modulus $\beta$ and the thickness quotient relating the patches thickness to the beam thickness for the pinned-pinned beam has been determined and presented in Figure 12 . 


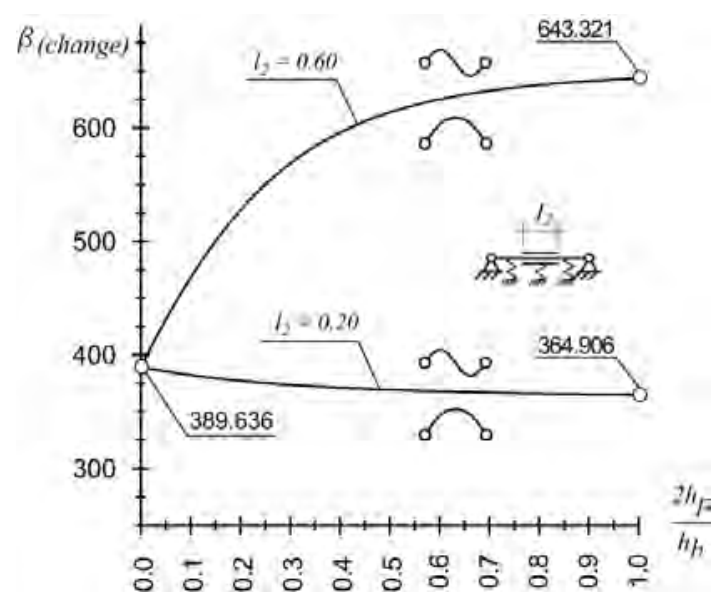

Fig. 12. The change in the first buckling modes for different Winkler foundation modulus and piezo patches to beam thickness quotient (PP beam)

The change in the first buckling mode occurs for different Winkler foundation modulus dependently on geometrical parameters of the system. For the longer piezosegment $\left(l_{2}=0.60\right)$ with growing values of the thickness ratio, a threshold curve separating the regions in which the first buckling load occurs for a zero-node mode and for a one-node mode, stretches out above the threshold curve for the shorter piezosegment $\left(l_{2}=0.20\right)$. The course of the curve for $l_{2}=0.60$ shows that when piezo patches become thicker, modes' transformation appears for greater values of the foundation modulus, whereas the curve sketched for $l_{2}=0.20$ exhibit a decreasing tendency which manifests that the first one-node mode exists for smaller values of the Winkler modulus.

\section{Conclusions}

The problem of critical buckling load for the beam with two piezoceramic layers resting on a Winkler elastic foundation has been studied. The obtained results concern the influence of support conditions, geometrical parameters and the piezoelectric actuation on the buckling capacity of the system. Regardless of the way of supporting, the greater the Winkler modulus and the longer the piezosegment, the higher critical buckling load of the system. Numerical calculation proves that a localisation of the piezopatches along the beam is also an influential factor with regard to its stability.

Application of the electric field to piezoceramics dependently on the direction of electric field vector generates an additional compressing or tensile axial force along the beam which modifies its buckling resistance. It has been numerically proved that the smaller the foundation modulus, the greater the influence of piezoactuation on the buckling capacity of the system. 


\section{References}

[1] Kerr A.D., Elastic and viscoelastic foundation models, Journal of Applied Mechanics 1964, 31, 491-498.

[2] Jančo R., Numerical and exact solution of buckling load for beam on elastic foundation, Transactions of the VŠB - Technical University of Ostrava, Mechanical Series 2013, 59, 21-26.

[3] Wang C.M., Wang C.Y., Reddy J.N., Exact Solutions for Buckling of Structural Members, CRC Press, Florida 2005.

[4] Glabisz W., Stability regions for a cantilever bar under independent potential and non-potential loading, Journal of Theoretical and Applied Mechanics 1981, 173-188.

[5] Dudzik A., Obara P., Stability analysis of Timoshenko beam resting on an elastic foundation, Acta Scientarum Polonorum, Architectura 2010, 9, 1, 17-30.

[6] Fazelzadeh S.A., Kazemi-Lari M.A., Stability analysis of partially loaded Leipholz column carrying a lumped mass and resting on elastic foundation, Journal of Sound and Vibration 2012, 332, 595-607.

[7] Eryilmaz A., Atay M.T., Coşkun S.B., Başbük M., Buckling of Euler columns with a continuous elastic restraint via Homotopy Analysis Method, Journal of Applied Mathematics 2012, 2013, 1-8.

[8] Przybylski J., Stability of an articulated column with two collocated piezoelectric actuators, Engineering Structures 2008, 30, 3739-3750.

[9] Przybylski J., Statics and dynamics problems of slender mechanical systems with integrated piezoceramic elements, Wydawnictwo Politechniki Częstochowskiej, Częstochowa 2012.

[10] www. annon-piezo.com/PZTMATERIALS.asp

[11] Hetenyi M., Beams on Elastic Foundation, Ann Arbor, University of Michigan Press, Michigan 1946. 